\title{
Sistematização da Assistência de Enfermagem ao paciente com câncer de pênis
}

\author{
Giulianna Soares GARCIA ${ }^{1}$ \\ Ana Helena Aquino PALMEIRA ${ }^{2}$ \\ Jeferson Barbosa SILVA ${ }^{3}$ \\ Jordânia Calixto da SILVA
}

\section{Recebido em: 28/05/2013 - Aprovado em: 30/07/2013 - Disponibilizado em: 15/08/2013}

\begin{abstract}
Resumo: O câncer de pênis se encaixa no grupo das mais antigas neoplasias conhecidas. Por ser física e psicologicamente mutilante, e por provocar resultados terapêuticos insatisfatórios, esta doença é tida como uma das mais perigosas que acometem o homem. Neste sentido, este estudo visa ampliar o conhecimento sobre o câncer de pênis e elencar diagnósticos e intervenções de enfermagem para a prestação de um cuidado holístico e humanizado ao paciente acometido por esta enfermidade. Trata-se de uma revisão da literatura nacional realizada por meio de consulta ao Scientific Electronic Library Online (Scielo) e à Biblioteca Virtual em Saúde (BVS). Foram consultados artigos de importância histórica até publicações do ano de 2012. Poucas são as literaturas que abordam aspectos relevantes acerca do câncer de pênis; sendo assim, cabe aos profissionais de saúde atuantes na área ampliarem os estudos acerca da temática para que novas estratégias de prevenção sejam desenvolvidas.
\end{abstract}

Descritores: Câncer; Pênis; Enfermagem.

\section{Systematization of Nursing Care for patients with penile cancer}

\begin{abstract}
Penile cancer (cancer of the penis) is in the group of the oldest known malignancies. Being physically and psychologically crippling, and cause of unsatisfactory therapeutic results, this disease is considered one of the most dangerous to affect men. Thus, this study aims to increase knowledge about penile cancer and rank diagnoses and nursing interventions to provide a holistic and humanized care for patients affected by this disease. This is a review of the national literature, developed by consulting the Scientific Electronic Library Online (SciELO) and the Virtual Health Library (VHL). Were consulted from articles of historical relevance to publications of the year 2012. There are a small number of literatures which discuss relevant aspects about penile cancer, so it is up to health professionals working on this area, to expand studies on this subject, so that new prevention strategies are developed.
\end{abstract}

Descriptors: Cancer; Penis; Nursing.

\footnotetext{
${ }^{1}$ Discente do Curso de bacharelado em Enfermagem pela Universidade Federal de Campina Grande - UFCG. E-mail: giulianna.garcia@hotmail.com;

${ }^{2}$ Discente do curso de bacharelado em enfermagem pela Universidade Federal do Rio Grande do Norte - UFRN. Email: palmeira.ana@hotmail.com;

${ }^{3}$ Bacharel em Enfermagem pela Universidade Federal de Campina Grande - UFCG. Especialista em Saúde Mental e Atenção Psicossocial em Dependência química pelas Faculdades Integradas de Patos - FIP. Membro do Grupo de Estudos e Pesquisas em História Oral e Saúde da Mulher - GEPHOSM da Universidade Federal da Paraíba - UFPB. Email: jefersonbarbosa_@hotmail.com;

${ }^{4}$ Bacharel em Enfermagem pela Faculdade de Ciências Médicas - FCM Campus Campina Grande. Especialista em Saúde da Família pelas Faculdades Integradas de Patos - FIP. E-mail: jordaniacalixto@msn.com
} 


\section{INTRODUÇÃO}

Câncer trata-se de uma malignidade envolvendo crescimento desordenado de células, as quais, com a invasão de tecidos e órgãos, levam ao desenvolvimento de tumores ou neoplasias malignas. Devido à série de divisões agressivas e incoercíveis que sofrem, estas células malignas podem disseminar-se para outras áreas do corpo (BRASIL, 2011).

O câncer constitui-se um problema significante de saúde pública, pois mata cerca de sete milhões de pessoas a cada ano. Até o ano de 2020, calcula-se que mais de dez milhões de pessoas serão acometidas por essa malignidade (BRAGA; DUTRA; OLIVEIRA, 2011).

No Brasil, a distribuição epidemiológica do câncer engloba o aumento das taxas de ocorrência dos tumores relacionados ao elevado status sócioeconômico (tais como câncer de mama, cólon, reto e próstata) e dos tumores associados com a pobreza, a saber: câncer de pênis, estômago, cavidade oral e colo de útero (GUERRA; GALLO; MENDONÇA, 2005).

O câncer de pênis se encaixa no grupo das mais antigas neoplasias conhecidas. Por ser física e psicologicamente mutilante, e por provocar resultados terapêuticos insatisfatórios, esta doença é tida como uma das mais perigosas que acometem o homem (REIS et al. 2010).

Segundo o Instituto Nacional do Câncer (INCA), os sinais e sintomas comumente encontrados no câncer de pênis são: ferida ou úlcera de difícil cicatrização, ou tumores encontrados na glande, prepúcio ou corpo do pênis; associada a estes, a presença de esmegma (secreção branca), sangramento e mau cheiro pode ser indicativo de câncer de pênis. Além disso, o aparecimento de ínguas na virilha pode indicar progressão da doença ou metástase (BRASIL, 2011).

Poucas publicações a respeito do câncer de pênis existem na literatura internacional, e grande parte delas advém de instituições isoladas, o que reflete a baixa ocorrência desta doença em países ricos. Sendo assim, questões importantes relacionadas a esta patologia necessitam de estudos mais aprofundados (PAULA et al. 2005).

Nesta perspectiva, este estudo visa ampliar o conhecimento sobre o câncer de pênis, favorecendo a reflexão sobre a importância de uma maior atenção à saúde do homem e a formulação de ações de prevenção para este tipo de câncer, especialmente nas regiões onde esta doença constitui-se um importante problema de saúde pública. Além disso, objetiva elencar diagnósticos e intervenções de enfermagem para a prestação de um cuidado holístico e humanizado ao paciente acometido por esta enfermidade.

\section{METODOLOGIA}

Trata-se de uma revisão de literatura nacional. Para tanto, considerou-se, como critérios de inclusão, estudos que abordassem o câncer de pênis, não havendo restrição quanto ao delineamento metodológico do estudo. Sendo assim, foram incluídos artigos de importância histórica até publicações do ano de 2012.

As buscas foram realizadas por cinco autores nas bases de dados: Scientific Electronic Library Online (Scielo) e Biblioteca Virtual em Saúde (BVS), através das seguintes palavras-chave encontradas nos 
Descritores em Ciências da Saúde (DeCS): câncer; pênis; enfermagem.

Após combinação dos descritores, foram encontrados 80 títulos, sendo selecionados apenas 13 , pois os demais não se enquadravam nos critérios de inclusão estabelecidos e nem na temática.

As informações foram analisadas e categorizadas em: aspectos da epidemiologia, etiologia, características histopatológicas, prevenção, estadiamento, diagnóstico, tratamento do câncer de pênis e sistematização da assistência de enfermagem.

\section{RESULTADOS E DISCUSSÃO}

\section{Epidemiologia}

A neoplasia de pênis é de ocorrência rara em países desenvolvidos, representando menos de $1 \%$ dos cânceres que acometem o homem (BARROS; MELO, 2009). A alta incidência de casos é percebida nos países em desenvolvimento, incluindo o Brasil, especialmente nas áreas mais carentes (REIS, 2010).

Nos Estados Unidos e na Europa, a incidência deste câncer não vai além de dois casos por 100.000 habitantes. Já na África, chega a representar $17 \%$ de todas as neoplasias malignas, correspondendo ao primeiro lugar do ranking mundial da doença (MELO et al. 2009). No Brasil, segundo dados do INCA, o tumor representa $2 \%$ de todos os casos de câncer no homem, ocorrendo com maior frequência nas regiões Norte e Nordeste do que nas regiões Sul e Sudeste (BRASIL, 2011).

Afeta, na sua maioria, indivíduos acima dos 60 anos de idade; contudo, jovens também podem ser acometidos, fato este confirmado pela ocorrência de $22 \%$ de casos registrados em pacientes com idade inferior a 40 anos (REIS, 2010).

Estudos revelam que existe uma tendência maior de incidência na raça negra, e que os homens casados identificam mais rapidamente a neoplasia de pênis em estádios iniciais do que os solteiros. Quanto ao perfil sócio-econômico-cultural dos acometidos, observa-se que esta doença afeta, principalmente, homens de baixo nível social e de pouca instrução, e que a tardança na busca de atendimento médico também advém do baixo nível cultural dos pacientes, constituindo-se, assim, um prejuízo iminente no resultado do tratamento (PAULA et al. 2005).

\section{Etiologia}

São diversos os fatores de risco e causas associados ao câncer de pênis, contudo a etiologia da neoplasia continua incerta (PAULA et al. 2005). Estudos apontam que a presença de fimose, doenças sexualmente transmissíveis, hábitos inadequados de higiene, baixa renda familiar e baixa escolaridade constituem-se os fatores de risco para esta doença (BARROS; MELO, 2009). É oportuno destacar que a associação de 30 a $50 \%$ do papilomavírus humano (HPV) ao carcinoma de pênis, mesmo sem a obtenção de provas exatas quanto a isto (GIL et al. 2001).

Quanto à presença de esmegma, nenhum carcinógeno específico foi encontrado ainda no mesmo; porém a ausência de circuncisão associada à má higienização e infecção pelo Mycobacterium smegmatis podem favorecer o surgimento do câncer de pênis, devido à irritação crônica do epitélio (PAULA et al. 2005). 
Outros fatores de risco incluem: condições inflamatórias crônicas (como balanopostites e líquen escleroso e atrófico); tabagismo; e fotoquimioterapia com UVA (raios ultravioletas A) ou PUVA, que corresponde aos raios ultravioletas A e psoralênico (CARVALHO, 2010).

\section{Histopatologia}

Cerca de $80 \%$ dos tumores penianos originam-se na glande, $15 \%$ no prepúcio e $5 \%$ no sulco coronal; contudo, nos casos clinicamente mais avançados, pode acometer mais de um local. Os tumores na glande costumam ser epidermóides, de alto grau e de profunda infiltração, diferentemente dos tumores prepuciais. Neoplasias originárias da haste peniana são incomuns (SOCIEDADE BRASILEIRA DE UROLOGIA a, 2006).

O câncer de pênis tem progressão loco-regional e desenvolvimento insidioso; cerca de $70 \%$ dos pacientes possuem a doença restrita ao pênis, $20 \%$ têm acometimento dos linfonodos inguinais e 10\% apresentam metástases (POMPEO; GIL, 2011).

Segundo suas características histopatológicas, os tumores penianos são classificados em: carcinoma epidermóide (mais freqüente - 95\%); carcinoma basalóide; carcinoma verrucoso; carcinoma sarcomatoso; carcinoma epidermóide da variante verrucosa; e carcinoma papilar (POMPEO; GIL, 2011).

O carcinoma verrucoso é tido como uma variante do carcinoma epidermóide, porém, ao contrário deste, apresenta menos embolização linfática, metástase linfonodal e caráter infiltrativo do corpo cavernoso; já os carcinomas basalóides e sarcomatóides possuem elevada taxa de metástases e mortalidade, pois são agressivos e de alto grau (SOCIEDADE BRASILEIRA DE
UROLOGIA b, 2006). Constituem-se tipos raros o carcinoma basalóide, sarcoma de Kaposi, melanoma e linfoma (PAULA et al. 2005).

\section{Prevenção}

Considerando-se os fatores de risco do câncer de pênis, é possível elencar, como medidas de prevenção: a circuncisão; a adoção de hábitos de higiene; o uso de preservativo durante a relação sexual; boa escolaridade; e condição socioeconômica favorável (BARROS; MELO, 2009).

De acordo com estudos realizados, a circuncisão é uma prática que deve ser estimulada devido à sua eficácia em impedir a gênese do câncer de pênis; associada a ela, a higienização eficiente do órgão (que deve ser realizada diariamente, principalmente na glande, em especial após masturbação ou relações sexuais) diminui drasticamente a incidência desta doença. É importante destacar que a circuncisão protege o pênis do câncer localizado e invasivo, porém não o protege do carcinoma in situ (SOUZA et al. 2011).

\section{Estadiamento}

De acordo com o INCA, estadiar o câncer compreende a avaliação do seu nível de disseminação. Isto se faz importante, pois se sabe que, quando a enfermidade encontrase apenas no órgão, as chances de sobrevivência são muito maiores. Para que se realize o estadiamento do câncer, são utilizadas regras estabelecidas internacionalmente; o sistema mais empregado é o Sistema TNM de Classificação dos Tumores Malignos (BRASIL, 2011).

O estádio TMN de 1978 avaliava o tamanho em centímetros da lesão peniana, 
comprometimento inguinal e presença ou não de metástases. Entretanto, a União Internacional contra o Câncer - UICC- fez um novo estádio, o TNM 2004, onde se analisa a dimensão em relação à profundidade de invasão - e não ao tamanho em centímetros-, e, igualmente a situação dos linfonodos e a presença de metástases (BRASIL, 2011; PAULA et al. 2005).

Ainda segundo o INCA, os parâmetros de estadiamento devem abranger a dosagem de marcadores tumorais e o estado funcional do paciente (BRASIL, 2011).

Vale destacar que, no carcinoma epidermóide de pênis, a disseminação ocorre, principalmente, por via linfática aos linfonodos inguinais - primeiramente os superficiais e, posteriormente, os profundos-. Metástases oriundas de disseminação hematogênica são raras (SOCIEDADE BRASILEIRA DE UROLOGIA b, 2006).

\section{Diagnóstico}

A fimose pode dificultar a visualização do tumor e, consequentemente, o seu diagnóstico. Portanto, qualquer lesão no pênis, associada à não retração do prepúcio, deve ser averiguada com suspeita de neoplasia (REIS, 2010).

A avaliação da extensão local é realizada através do exame físico, mas, caso não seja possível determiná-la, pode ser utilizada a ressonância magnética ou a ultrassonografia. Se for detectada a presença de linfonodos palpáveis, a biópsia por aspiração com agulha fina guiada por ultrassonografia pode ser empregada. Pode ser utilizada ainda a linfocintilografia injeção de rádio colóide em torno da lesão e avaliação da região inguinal com raios gama-, porém apresenta menor eficácia que a linfadenectomia regional, a qual tem efeito curativo em lesões mínimas e capacidade de estabelecer o estadiamento do câncer. Para a detecção de metástases, a radiografia de tórax, tomografia computadorizada de abdome e pelve e a tomografia por emissão de pósitrons constituem-se métodos diagnósticos eficientes (SOCIEDADE BRASILEIRA DE UROLOGIA b, 2006).

\section{Tratamento}

O tratamento do câncer de pênis objetiva a remoção completa do tumor através de diversas técnicas como postectomia, cirurgia micrográfica de Mohs, criocirurgia, radioterapia, ablação com laser de $\mathrm{CO} 2$ e penectomia e suas variações (MELO, 2009).

O mesmo é baseado na análise histopatológica da lesão, onde verifica-se a extensão do tumor primário e a sua classificação através da realização da biópsia. Antes da realização da biópsia, se dá início à terapia antibiótica e prossegue com a terapia cirúrgica, estendendo-se por quatro a seis semanas. Apenas com o diagnóstico do tecido confirmado, pode-se tratar, por meio de excisão cirúrgica local, quimioterapia ou terapia de radiação superficial, os tumores superficiais pequenos (REIS, 2010).

As amputações parciais ou totais do pênis correspondem às modalidades terapêuticas mais usadas na atualidade, com controle de mais de $90 \%$ da neoplasia. A radioterapia é utilizada apenas quando os pacientes se recusam a fazer a cirurgia, pois não dá o exato estadiamento do câncer e não controla a lesão local com eficácia (PAULA, 2005; POMPEO; GIL, 2011).

Segundo o INCA, se o câncer de pênis for identificado em sua fase precoce, poderá se evitar a amputação do pênis e a produção 
de seqüelas físicas, sexuais e psicológicas associadas a ela (BRASIL, 2011).

Assistência de enfermagem, segundo a taxonomia da North American Nursing Diagnosis Association (NANDA)

Os principais diagnósticos de Enfermagem que podem ser traçados para a assistência de um paciente acometido por câncer de pênis estão explicitados nos quadros abaixo (NORTH AMERICAN NURSING DIAGNOSIS ASSOCIATION, 2012), juntamente com as principais intervenções de enfermagem correspondentes, desenvolvidas segundo a Classificação de Intervenções de Enfermagem (NIC): 


\section{Diagnóstico de Enfermagem (NANDA)}

\section{Prescrições de} Enfermagem

\section{Domínio 12 (Conforto)}

Dor relacionada à ulceração peniana decorrente do processo

\section{Domínio 11 (Segurança e}

proteção)

Integridade da pele prejudicada relacionada à carcinogênese tissular carcinogênico

Controle da dor

\section{Cuidados com lesão}

\section{Proteção contra infecção}

Risco para infecção relacionado à exposição das lesões a microrganismos, secundário à condição patológica e/ou procedimento cirúrgico

\section{Domínio 3 (Eliminação e Troca)}

Eliminação urinária prejudicada relacionada à disseminação do carcinoma e acometimento uretral

\section{Intervenções de Enfermagem (NIC)}

- Realizar uma avaliação abrangente da dor (frequência, qualidade, intensidade, fatores precipitantes);

- Assegurar que o paciente receba cuidados precisos de analgesia, avaliando sua eficácia junto ao mesmo;

- Reduzir ou eliminar fatores que precipitem ou aumentem a experiência de dor (p. ex.: medo, fadiga, fatores ambientais que influenciem no desconforto);

- Monitorar características da lesão (grau de comprometimento tissular, aspecto, odor, exsudato); - Monitorar cicatrização da ferida;

\section{- Proceder com a limpeza da lesão e aplicação de curativo adequado ao tipo de ferida. \\ - Discutir com o paciente sobre métodos não farmacológicos para alívio da dor (p. ex.: relaxamento, musicoterapia, acupressão, etc).}

Controle de Infecção

Proteção contra infecção

Controle da eliminação urinária
- Monitorar SSVV e examinar a
pele/lesão em busca de sinais
flogísticos;

- Manter hábitos de assepsia, orientando paciente e família sobre sinais de infecção e os modos de preveni-la.

- Promover prática de técnicas adequadas de assepsia e lavagem das mãos, orientando funcionários, familiares, pacientes e visitantes;

- Limpar pele do paciente com agente antibacteriano, quando adequado;

- Assegurar emprego de técnica adequada no manuseio de feridas, bem como na inserção e manuseio do cateter vesical.

- Monitorar SSVV e examinar a pele/lesão em busca de sinais flogísticos;

- Monitorar eliminação urinária (frequiência, odor, aspecto sinais ou sintomas de retenção urinária e débito urinário);
- Manter hábitos de assepsia, orientando paciente e família sobre sinais de infecção e os modos de preveni-la.

- Proceder com cateterismo vesical (demora ou alívio), quando adequado;

- Aplicar medidas de controle da dor. 


\section{Domínio 5}

\section{(Percepção/Cognição)}

Déficit de conhecimento sobre a doença, procedimentos e exames diagnóstico

\section{Domínio 9 (Enfrentamento/ tolerância ao estresse)}

Medo e enfrentamento ineficaz relacionados com o diagnóstico de câncer de pênis, seu prognóstico e tratamento

\section{Ensino: Processo de Doença}

Facilitação da aprendizagem

Melhora do enfrentamento

Grupo de apoio

Distração

Aconselhamento sexual

Disfunção sexual relacionada aos efeitos da terapia: cirurgia, radioterapia, quimioterapia

\section{Domínio 6 (Auto percepção)}

Distúrbio da autoimagem corporal relacionado às injúrias e/ou procedimento cirúrgico no membro acometido

Melhora da imagem corporal
- Avaliar atua nível de conhecimento do paciente em relação ao processo de doença; - Oferecer informações ao paciente sobre sua condição, descrevendo sinais e sintomas comuns da doença, quando adequado;

- Adaptar instrução ao nível de conhecimento e compreensão;

- Auxiliar paciente a identificar metas a curto e longo prazo;

- Usar abordagem calma e segura, auxiliando o paciente em seu luto e perdas/incapacitações decorrentes da doença;

- Usar um grupo de apoio durante os estágios de transição, para auxiliar o indivíduo a adaptar-se a um novo modo de vida;

- Encorajar o indivíduo a escolher técnica (s) de distração desejada (s), orientando sobre os benefícios da estimulação de vários sentidos.

- Discutir efeitos da doença sobre a sexualidade;

-Incluir cônjuge no aconselhamento, quando adequado, assegurando sempre a privacidade do cliente;

Escutar ativamente
- Orientar antecipadamente para preparar o paciente para mudanças na imagem corporal; impacto de qualquer desfiguramento, como uso de roupas ou ações que melhorem a aparência; - Identificar meios de reduzir
-Discutir formas alternativas de expressão sexual;

Encaminhar paciente para adequado

- Auxiliar paciente a separar aparência física dos sentimentos de valor pessoal, quando adequado. 



\section{CONCLUSÃO}

Poucas são as literaturas que abordam aspectos relevantes acerca do câncer de pênis, que, como foi visto, acarreta uma série de prejuízos ao indivíduo.

Para muitos homens, a realização da penectomia implica na perda da essência e da virilidade, e assim ocorre a gênese da baixa autoestima e prejuízo de suas vidas sexual, afetiva e social. Por esta razão, é de extrema importância que sejam desenvolvidas medidas de incentivo aos homens para efetivação da procura dos serviços de saúde, com o objetivo de torná-los protagonistas do cuidado, inspirando a responsabilização daqueles na manutenção e/ou recuperação de sua saúde sexual. Além disso, cabe aos enfermeiros atuantes na área ampliarem os estudos acerca da temática para que novas estratégias de prevenção sejam desenvolvidas.

\section{REFERÊNCIAS}

1. BARROS, É.N.; MELO, M.C.B. Câncer de pênis: perfil sóciodemográfico e respostas emocionais à penectomia em pacientes atendidos no Serviço de Psicologia do Hospital de Câncer de Pernambuco. Rev. SBPH [online]. vol.12, n.1, p. 99-111, 2009. Disponível em: http://pepsic.bvsalud.org/scielo.php?pi $\mathrm{d}=\mathrm{S} 1516$ -

$\underline{08582009000100008 \& \text { script }=\text { sci_artte }}$ xt\&tlng=es. Acesso em 5 de junho de 2011.
2. BRAGA, R.O.B.; DUTRA, D.A; OLIVEIRA, M.C.M. Incidência Geopatológica de Neoplasia no Brasil. Revista Inspirar - movimento e saúde. Vol. 3, n. 5, set/out de 2011. Disponível em: http://www.inspirar.com.br/revista/wp content/artigo_10_173_revista_cientifi ca_edi\%C3\%A7\%C3\%A3o_1.pdf

3. BRASIL. Instituto Nacional do Câncer 2011. Disponível em: http://www.inca.gov.br/. Acesso em 5 de junho de 2011.

4. CARVALHO, N.S. et al. Associação entre HPV e câncer peniano: revisão da literatura; DST - Jornal brasileiro de Doenças Sexualmente Transmissíveis. vol. 19, n.2, p. 92-95. 2007. Disponível em: http://www.dst.uff.br/revista19-22007/6.pdf. Acesso em 5 de junho 2011.

5. GIL, A.O. et al. Analysis of the association between human papillomavirus with penile carcinoma; International Braz J Urol. Vol. 27, p. 461-468. 2001. Disponível em: http://www.brazjurol.com.br/outubro 2001/Gil 461_468.htm. Acesso em 5 de junho de 2011.

6. GUERRA, M.R.; GALLO, C.V.M.; MENDONÇA, G.A.S. Risco de câncer no Brasil: tendências e estudos epidemiológicos mais recentes; Revista Brasileira de Cancerologia. Vol. 51, n.3, p. 227-234; 2005. Disponível em: http://www.eteavare.com.br/arquivos/ 81_392.pdf. Acesso em 5 de junho de 2011. 
7. MELO, É.L. et al. Linfadenectomia inguinal no câncer de pênis: há como diminuir a morbimortalidade do procedimento?; Sinopse de Urologia. Vol.13, n. 2, Set 2009. Disponível em: http://www.moreirajr.com.br/revistas. asp?fase $=$ r003\&id_materia $=4181$. Acesso em 5 de junho de 2011.

8. NORTH AMERICAN NURSING DIAGNOSIS ASSOCIATION. Diagnósticos de enfermagem da NANDA: definições e classificação. 2012-2014. Porto Alegre: Artmed, 2012. 606 p.

9. PAULA, A.A.P. et al. Carcinoma epidermóide do pênis: considerações epidemiológicas, histopatológicas, influência viral e tratamento cirúrgico; Revista Brasileira de Cancerologia vol. 51, n.3, p. 243-252. 2005. Disponível em: http://www.inca.gov.br/rbc/n_51/v03/ pdf/revisao3.pdf. Acesso em 5 de junho de 2011.

10. POMPEO, A.C.L.; GIL, A.O. Câncer de Pênis. 2011. Disponível em: http://www.hpvinfo.com.br/hpv13.htm. Acesso em 14 de outubro de 2012

11. REIS, A.A.S. et al. Aspectos clínicoepidemiológicos associados ao câncer de pênis; Ciência \& Saúde Coletiva, vol. 15(Supl.1), p. 1105-1111, 2010. Disponível em: http://www.scielo.br/pdf/csc/v15s1/01 8.pdf. Acesso em 5 de junho de 2011.

12. SOCIEDADE BRASILEIRA DE UROLOGIA a; Carcinoma do Pênis Parte I; Associação Médica Brasileira e Conselho Federal de
Medicina; 2006; 18 páginas. Disponível em: http://www.projetodiretrizes.org.br/6 volume/12-CarcinomaPenisParte1.pdf. Acesso em 5 de junho de 2011.

13. SOCIEDADE BRASILEIRA DE UROLOGIA b; Carcinoma do Pênis Parte II; Associação Médica Brasileira e Conselho Federal de Medicina; 2006; 9 páginas. Disponível em: http://www.projetodiretrizes.org.br/6 volume/13-

CarcinomaPenisParteII.pdf. Acesso em 5 de junho de 2011.

14. SOUZA, K.W. et al. Estratégias de prevenção para câncer de testículo e pênis: revisão integrativa; Rev. Esc. Enferm. USP. Vol. 45, n.1, p. 277-82, 2011. Disponível em: http://www.scielo.br/pdf/reeusp/v45n1 139.pdf. Acesso em 5 de junho de 2011.

15. DOCHTERMAN, J.M.; BULECHEK, G.M. Classificação das Intervenções de Enfermagem (NIC). , 4. ed. Porto Alegre: Artmed, 2008. 